\title{
Direct sensitivity analysis to nuclear data of thorium molten salt reactors at equilibrium using MURE
}

\author{
A. Bidaud ${ }^{1, a}$, O. Meplan ${ }^{2}$, A. Nuttin ${ }^{1}$, S. David ${ }^{2}$, J.N. Wilson ${ }^{2}$, F. Michel-Sendis ${ }^{2}$, P. Guillemin ${ }^{1}$, and N. Capellan ${ }^{2}$ \\ ${ }^{1}$ LPSC, Université Joseph Fourier Grenoble 1, CNRS/IN2P3, Institut National Polytechnique de Grenoble, France \\ 2 Institut de Physique Nucléaire d'Orsay, IN2P3/CNRS, 91406 Orsay, France
}

\begin{abstract}
In this paper we propose to use the MURE (MCNP Utility for Reactor Evolution) package to calculate directly nuclear data sensitivities of the breeding gain of a thorium molten salt reactor. The continuous fuel reprocessing is used to control reactivity. This control coupled with the fact that the fuel has reached its equilibrium, induces feedback effects on nuclear data sensitivities. That is why sensitivities are calculated directly by recalculating the fuel equilibrium of a simplified model after modifications of the ACE files for the reactions that are expected to have the strongest contribution to the uncertainty: 233U capture cross section and neutron fission yield.
\end{abstract}

\section{Introduction}

\subsection{Thorium molten salt reactors}

Molten Salt Reactors (MSR) are one of the six Gen IV reactors. In these concepts, the fuel is dissolved in the molten salt, which is used also as coolant.

This configuration has proven to be both very efficient and safe in successful experiments done in Oak Ridge during the 60's [1]. Furthermore, the constant circulation of the liquid fuel allows on-line recycling. In thorium cycle, the regeneration is limited by the quite low number of neutron available. Thus, the continuous removal of the poisoning fission products makes the regeneration feasible. This concept is being developed [2] in France in the framework of the GEDEPEON research group. The sensitivity and uncertainty analysis of the $\mathrm{k}_{\mathrm{eff}}$ of one of those concepts has been studied in details $[3,4]$. In the configuration chosen for this paper, the neutron spectrum is epi-thermal and thus quite sensitive to the nuclear data of the resonance range.

\subsection{Concept versatility}

Historically, molten salt circulates in a hexagonal lattice of graphite channels. By changing the hexagon sides or the channel diameters, the neutron spectra and then the neutronic properties can be changed in a very wide range, from well thermalized spectrum in the Molten Salt Breeder Reactor [5] (Oak Ridge's most advanced reactor designed in the 70's) to epi-thermal and even fast spectrum when the graphite is removed. If thermal reactors used to be favoured because of the low fissile inventory of ${ }^{233} \mathrm{U}$ needed at start-up, fast reactors are currently being investigated in details [6]. A fast spectrum MSR benefits not only of the high number of neutrons available for breeding and transmutation as any other fast reactor concepts, but also of strong negative coefficients that are hardly achievable by reactors using other coolants.

${ }^{a}$ Presenting author, e-mail: bidaud@lpsc.in2p3.fr

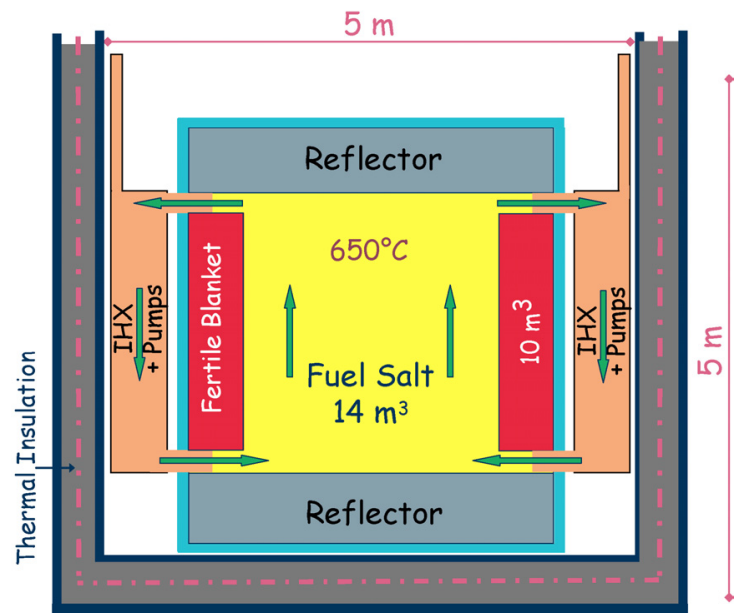

Fig. 1. Full scale model of molten salt reactors.

\section{Previous uncertainty analysis}

Previous results of sensitivity and uncertainty analysis have already been published for an epi-thermal system and are reminded shortly in this part. The objective of this paper is to present the tools developed to confirm these results. In the future, we expect to expand the study to other spectra range using all the tools available.

\subsection{Uncertainty on $\mathbf{k}_{\mathrm{eff}}$}

$\mathrm{K}_{\mathrm{eff}}$ sensitivity and uncertainty analysis based on a classical perturbation theory approach had shown [4] that thorium capture cross section used to be responsible for the biggest part of the total uncertainty that was estimated to about $4000 \mathrm{pcm}$. Updated results [7] based on recent covariance matrices evaluated in the scope of AIEA CRP on Evaluated Nuclear Data for Thorium-Uranium Fuel Cycle [8], themselves based on new experimental data [9], has shown that the contribution of Thorium cross section uncertainties has been largely reduces down to $800 \mathrm{pcm}$ which is more or less comparable to the 


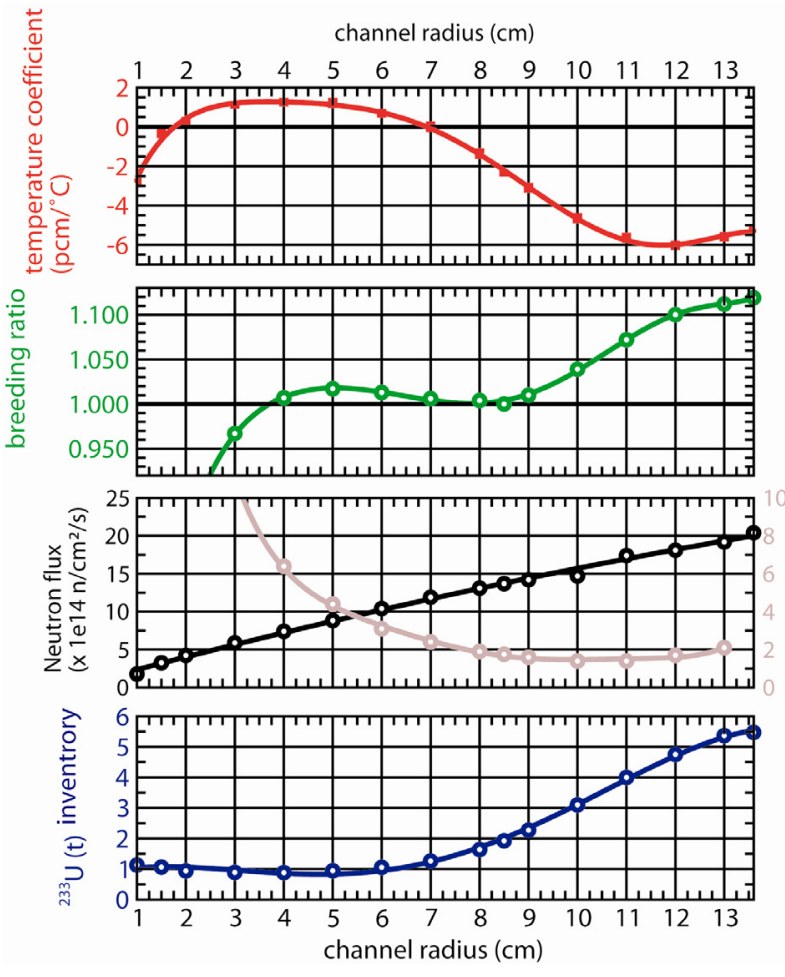

Fig. 2. Evolution of main neutronics parameters with channel diameter.

contributions of ${ }^{233} \mathrm{U}$ and ${ }^{234} \mathrm{U}$ nuclear data uncertainties. This important improvement makes MSR developer quite confident in the reliability of the nuclear data. Furthermore it demonstrates both the quality of the work achieved by the combination of recent nuclear experiment with recent nuclear data evaluation tools and the necessity for covariance matrix evaluation, in order to convince nuclear data users of the reality of the improvement.

\subsection{Breeding gain uncertainty}

Breeding gain (BG) is the most important parameter to evaluate the long term development capacity of next generation reactors. Non-breeder reactors cannot win market shares as they will eventually fall short of fissile material.

In a critical reactor, there are no uncertainties on $\mathrm{k}_{\mathrm{eff}}$ : it is equal to one, by definition of criticality. This means that the burden of nuclear data uncertainty is not carried by the $\mathrm{k}_{\text {eff }}$ but by ${ }^{233} \mathrm{U}$ proportion because this proportion is changed in a MSR to keep the reactor critical. This kind of error compensation is expected [5] to have a big impact on the BG uncertainty analysis.

In a MSR, the BG can be defined as the ratio of ${ }^{233} \mathrm{U}$ production divided by ${ }^{233} \mathrm{U}$ consumption:

$$
B G=\frac{\Sigma_{c}^{T h}-\Sigma_{c}^{233} \mathrm{~Pa}}{\Sigma_{f}^{233} \mathrm{U}+\Sigma_{c}^{233} \mathrm{U}} .
$$

On the one hand, the sensitivity of BG to Thorium capture cross section is expected to be extremely important. On the other hand, ${ }^{233} \mathrm{U}$ fission cross section and Th cross section have symmetric positions and these positions are inversed to their positions in the definition of $\mathrm{k}_{\mathrm{eff}}$. This inversion is the source of the error compensation.

After some years, the isotopes proportions in the fuel are expected to reach their equilibrium. This should be another source of error compensations.

The effect of the equilibrium can be studied using the D factors [10] which count the number of neutrons produced by the destruction of one heavy nuclide by fission. The effect of the equilibrium has been found [4] to bring only minor corrections to the following approximation of the $\mathrm{BG}$ in a critical reactor:

$$
B G=v-2 \times(1+\alpha) .
$$

Where $v$ is the fission neutron yield and $\alpha$ is the fission to capture ratio of the main fissile material ${ }^{233} \mathrm{U}$.

If the control of the criticality and the equilibrium can reduce the sensitivities of BG to some cross sections such as fertile nucleus' capture cross sections, they can also increase the sensitivities of others such as fissile nucleus's capture cross sections. $80 \%$ of all the fissions happen on ${ }^{233} \mathrm{U}$. If its capture cross section uncertainty reaches the $10 \%$ as proposed by G. Palmiotti et al. [10], the uncertainty on the BG could be as high as $4 \%$, which reduces a lot the reliability of any numerical simulation. This paper aim is to confirm the high sensitivity of $\mathrm{BG}$ to ${ }^{233} \mathrm{U}$ capture cross section, due to the $\mathrm{k}_{\mathrm{eff}}$ control system and the reach of the fuel equilibrium.

\section{MURE}

The main aim of the MURE package is to perform nuclear reactor time-evolution using the widely used particle transport code MCNP [12], a Monte Carlo code that is mostly written in FORTRAN. MURE [13] is based on $\mathrm{C}++$ objects allowing a great flexibility in the use. There are three main parts in this library:

- Definition of the geometry, materials, neutron source, tallies. It can be used independently of the two others; it allows "easy" generation of MCNP input files by providing a set of classes for describing complex geometries such as lattices.

- Construction of the nuclear tree, the network of links between neighbouring nuclei via radioactive decays and nuclear reactions. Nuclei with half-lives which are very much shorter than the evolution time steps are removed from the tree and their parents and daughters re-linked in the correct way.

- Evolution of some materials, by solving the corresponding Bateman's equations. The evolution of the fuel within a given reactor over a time period of up to several years is simulated by successive steps of MCNP calculation and numerical integration of Bateman's equations. Each time MCNP is called, the reactor fuel composition will have changed due to the reaction/decay process occurring inside.

Moreover an interface to NJOY [14] in order to process crosssections at the wanted temperature is provided. 


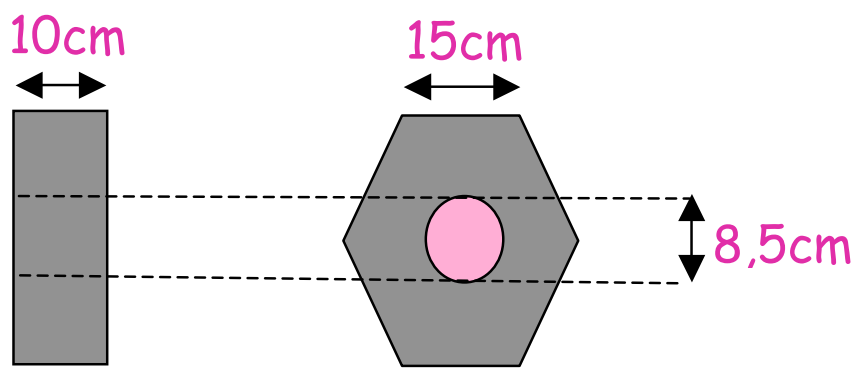

Fig. 3. Cell scale model of molten salt reactors.

\subsection{Implementation of constant reprocessing}

The continuous removal of fission product and other nuclei in the salt is in competition with nuclear reactions and decays in the fuel evolution. Thus, it must be taken into account at each time steps, this forces the implementation of specific methods needed to:

- Extract selected nuclei in function of their $\mathrm{Z}$ that characterize their chemical activity and in function of the reprocessing speed that is dependant of the extraction mode: bubbling or pyroprocessing.

- Control the criticality by adjusting the ${ }^{233} \mathrm{U}$ proportion. The weight of ${ }^{233} \mathrm{U}$ is estimated roughly on the base of its contribution to fissions and absorptions. The amount of ${ }^{233} \mathrm{U}$ to be added is then calculated so as to maintain the $\mathrm{k}_{\mathrm{eff}}$ equal to the initial $\mathrm{k}_{\mathrm{eff}}$.

- Control the amount of heavy nuclide by adjusting ${ }^{232} \mathrm{Th}$ proportion. This correction is needed to assure the chemical stability of the salt despite Fission product and actinide extraction.

- Simulate the evolution of the nuclei extracted from the core. MURE was designed to make the evolution of in core materials, now it can simulate the evolution of cells that are kept out of neutron flux.

The flux level is automatically adjusted by MURE so as to maintain the power constant.

\subsection{Model used for sensitivity calculation}

Our purpose is to estimate the sensitivities of the regeneration gain at equilibrium. This supposes to calculate the evolution toward equilibrium with modified sets of cross sections. Discrepancies between the BG at equilibrium divided by the changes in the cross sections give the sensitivities. This supposes that the discrepancies are far bigger than the stochastic uncertainty and thus long MCNP calculations are required. To save computation time, we have modelled only one channel of the graphite matrix with reflective boundary conditions. The $\mathrm{k}_{\mathrm{eff}}$ of our cell model fuelled with the same salt than the full scale critical reactor is $1,0618 \pm 60 \mathrm{pcm}$. For each equilibrium search, the $\mathrm{k}_{\mathrm{eff}}$ is forced to remain constant and equal to this value.

\subsection{Nuclear data modifications}

The importance of ${ }^{232} \mathrm{Th}$ and ${ }^{233} \mathrm{U}$ capture cross sections have been explained here. The sensitivities to these cross sections are evaluated after a $10 \%$ modification of their value. This $10 \%$ is slightly bigger than the estimated uncertainty on these cross sections in particular for ${ }^{232} \mathrm{Th}$ since it has been largely improved recently has written earlier. Nevertheless, this quite big modification makes the differences in results more important when compared to stochastic uncertainty and then reduces calculation time.

Table 1. Comparison of $\mathrm{k}_{\mathrm{eff}}$ and BG for the full core model and the cell model.

\begin{tabular}{lll}
\hline First & Full scale & Cell model \\
$\mathrm{k}_{\text {eff }}$ & 1.000 & 1.0624 \\
$\mathrm{BG}$ & 1.002 & 0.90465 \\
\hline
\end{tabular}

These modifications were done in the ACE files read by MCNP. Total cross sections were also modified to keep the set self-consistent. Moreover, the absorption cross section was modified accordingly to capture cross section in order to have the modifications taken into account not only by MURE in the evolution calculation but also by MCNP in the neutron balance, i.e., $\mathrm{k}_{\mathrm{eff}}$, calculation.

The number of neutron produced by fission is also a very important parameter, both for the criticality calculation but also for the BG calculation. The sensitivities are evaluated by a $1 \%$ increase of its value. The modification was done directly in the ENDF file before NJOY treatment. This modification was very easy to do because only a dozen of values are used to describe ${ }^{233} \mathrm{U}$ fission neutron yields.

\subsection{Direct sensitivity results}

Table 2 gives the results of the sensitivity analysis.

Table 2. Results of the sensitivity study. The $\mathrm{k}_{\mathrm{eff}}$ uncertainty given corresponds to the uncertainty coming from the stochastic uncertainty, not from the nuclear data uncertainty. ND (Nuclear Data) uncertainty is our estimate of the average uncertainty of the modified nuclear data on the spectrum relevant to this reactor concept given in pcm $\left(10^{-5}\right)$.

\begin{tabular}{lcccc}
\hline & Ref case & Th & $\mathrm{U}$ & $\mathrm{U}$ \\
\hline data & & $\sigma_{\text {capture }}$ & $\sigma_{\text {capture }}$ & $v$ \\
Change (\%) & & $10 \%$ & $10 \%$ & $1 \%$ \\
BG & 0.90465 & 0.91527 & 0.89195 & 0.92303 \\
Sensitivity $(\% / \%)$ & & 0.117 & -0.14 & 2.03 \\
ND uncertainty & & $4 \%$ & $10 \%$ & $1 \%$ \\
BG uncertainty & & 450 & 1400 & 2000 \\
$\mathrm{k}_{\text {eff }}$ unc. $(\mathrm{pcm})$ & 60 & 250 & 100 & 100 \\
\hline
\end{tabular}

As expected, the sensitivity to thorium capture cross section is the smallest which confirms the very efficient error compensation provided by the $\mathrm{k}_{\text {eff }}$ control. The uncertainty on this data is about $4 \%$ in a very broad spectrum. This means 
that the contribution of this data to the uncertainty on BG is the smallest of the ones estimated here. The sensitivity of uranium capture cross section is slightly smaller that the one expected [4] which was estimated without any perturbation tool. If one changes one cross section by $1 \%$, the reaction rate linked to this cross section will not change by $1 \%$ but a little less because of self shielding that will change the neutron flux. Perturbation Theory allows to take into account this indirect effect.

The sensitivity to neutron fission yield is very close to the one expected because no self-shielding effect can decrease the effect of the change on this nuclear data. Our estimation of the uncertainty on this data is probably slightly too big. Anyway, the contribution of this data to the total uncertainty is probably the biggest, of the order of $2000 \mathrm{pcm}$.

\section{Conclusions}

MURE is a versatile tool that allows the coupling of a reactor with its reprocessing facility as in a molten salt reactor.

The predominant roles of ${ }^{233} U$ capture cross section and fission neutron yields in the uncertainty of the breeding gain are confirmed by this direct sensitivity study.

Due to recent improvements in its accuracy, ${ }^{232} \mathrm{Th}$ capture cross section is not the dominant contributor the uncertainty, neither for the $\mathrm{k}_{\mathrm{eff}}$ nor for the BG.

On the base of the sensitivity found here, the total uncertainty on BG is expected to low as high as $2500 \mathrm{pcm}$ which is quite important as it is.
These preliminary results should be improved by using more statistics and extended to the whole range of neutron spectra that MSR could use.

The MURE development team and in particular its guru "pto" must be thanked for the quality of their programs.

\section{References}

1. P. Haubenreich, Nucl. Appl. Technol. 8, 118 (1970).

2. L. Mathieu, Ph.D. thesis, Institut Polytechnique de Grenoble, 2005.

3. A. Bidaud, in Proceedings of Workshop on Nuclear Data Needs for GEN IV Reactors, Antwerpen, Belgium, 2005, edited by P. Rullhusen (World Scientific, 2005), p. 101.

4. A. Bidaud, Ph.D. thesis, University of Paris XI, 2005.

5. Oak Ridge National Laboratory Report No. 4541, 1971.

6. E. Merle-Lucotte, in Proceedings of ICAPP Conference, Nice, 2007.

7. A. Bidaud, in Proceedings of PHYSOR Conference, Vancouver, 2006.

8. A. Trkov, in AIEA-CRP 2003.

9. G. Aerts, in Proceedings of Nuclear Data Conference, Santa Fe, 2004.

10. M. Salvatores, Nucl. Sci. Eng. 124, 280 (1996).

11. G. Palmiotti, in JEFF-DOC 1063rev1, 2005.

12. J.F. Briesmeister, Los Alamos National Laboratoy Report 12625 M, 1998.

13. O. Meplan, in Proceedings of European Nuclear Conference 2005.

14. B. Mac Farlane, in RSICC CODE PACKAGE PSR-368, 1998. 